\title{
Multi-Hamiltonian structures for r-matrix systems
}

\author{
J. Harnad and J.C. Hurtubise
}

CRM preprint 2850

\begin{abstract}
For the rational, elliptic and trigonometric r-matrices, we exhibit the links between three "levels" of Poisson spaces: (a) Some finite-dimensional spaces of matrix-valued holomorphic functions on the complex line; (b) Spaces of spectral curves and sheaves supported on them; (c) Symmetric products of a surface. We have, at each level, a linear space of compatible Poisson structures, and the maps relating the levels are Poisson. This leads in a natural way to Nijenhuis coordinates for these spaces. At level (b), there are Hamiltonian systems on these spaces which are integrable for each Poisson structure in the family, and which are such that the Lagrangian leaves are the intersections of the symplective leaves over the Poisson structures in the family. Specific examples include many of the well-known integrable systems.
\end{abstract}

\section{Introduction}

In this note, we show that a linear ("compatible") family of holomorphic Poisson brackets defined on certain subspaces of the loop algebra of $G l(r, \mathbb{C}$ ) ("level (a)") endows them with a multi-Hamiltonian structure (see, e.g. Magri [Mg1] ), and that it possesses a common set of Nijenhuis or Darboux-Nijenhuis coordinates (in a sense to be defined below). This family of brackets contains both the linear Lie Poisson structures on the algebra, as well as the quadratic structures due to Sklyanin [Sk1,2]. The brackets are all defined in terms of $r$-matrices, and we shall consider three cases, the rational, trigonometric and elliptic $r$-matrices.

This family of subspaces, with their accompanying families of brackets and Hamiltonians, is an important one, since it includes many, if not most, of the well-known cases of integrable systems: to cite some familiar examples, the Gaudin model, spin chains, the Toda lattice, the various tops (Euler, Lagrange, Manakov, Kovalevski, Steklov), the Landau Lifschitz equation, as well as the finite gap cases of the KdV, NLS, CNLS or Boussinesq hierarchies. References include the book [FT], the survey [RS2] and the references therein, or the articles [Mo, AvM, RS1, AHP, HHu].

In the three cases of rational, elliptic and trigonometric $r$-matrices, the phase spaces have geometric interpretations as spaces of meromorphic endomorphisms of a vector bundle over a compact Riemann surface. This bundle has the property that it is holomorphically rigid under deformation, at least if one fixes the top exterior power. Bundles with this property exist on the Riemann sphere (rational case), elliptic curves (elliptic case) and their nodal degenerations (trigonometric case). This interpretation will be crucial in the geometric study of the systems.

In all three cases, once one reduces by the action of the group of automorphisms of the bundle, one can describe the reduced phase space as a space of pairs (spectral curve, line bundle on the spectral curve) ("level (b)"). These spaces, in turn, have natural families

The authors of this article would like to thank NSERC and FCAR for their support 
of Poisson brackets on them, the Mukai brackets, which turn out to be identical to the (reduced) brackets of our family.

Furthermore, each of these spaces admits Poisson maps to a symmetric product of a surface equipped with a family of non-degenerate Poisson structures ("level(c)"). (More properly, one should consider a Hilbert scheme instead of a symmetric product). The point of this note is that all three levels possess linear families of Poisson structures, and these families can be identified in such a way that the maps relating the three levels are all Poisson, for any Poisson structure in the family. In particular, the surface of level (c) provides the Nijenhuis coordinates referred to above.

In short we will prove the following

THEOREM 1. For the rational, trigonometric and elliptic $r$-matrices, there are maps from

(a) A space $\mathcal{M}$ of, in the rational case, elements of the loop algebra on $G l(r, \mathbb{C})$, and in the trigonometric and elliptic case, of pairs (element of the loop algebra on $G l(r, \mathbb{C})$, point on a fixed nodal rational or elliptic curve)

(b) A space $\mathcal{N}$ of pairs (spectral curves $S$, torsion free sheaves $L$ on $S$ ), to

(c) The Hilbert scheme $H_{i l b}(T)$ of a surface $T$, where $g=$ genus $(S)$.

Each level posseses a linear family of Poisson structures, and the maps are Poisson for each of these structures. The maps from level (a) to (b) are quotients by a group of automorphisms. The maps from level (b) to (c) are generically immersions on symplectic leaves. At level (c), the Poisson structures are all non-degenerate on open sets, and there are natural coordinates which provide Nijenhuis coordinates for the system.

Precise definitions are given below. One has a natural set of Hamiltonians defined at level (a), given in terms of the characteristic polynomial of the matrices, and equivalently in terms of the coefficients of the equation of the spectral curve $S$. We will show

Theorem 2. The map $(S, L) \mapsto S$ defines an integrable system, that is a Lagrangian fibration, on the spaces $\mathcal{N}$, for each Poisson structure in the family at level (b). The Lagrangian leaves are cut out by fixing the values of the Casimirs for each structure in the family.

As one varies the Poisson structure, then, some of the Casimirs for one structure turn into "effective" Hamiltonians for another of the structures (i.e., giving actual flows), in such a way that the union over the Poisson structures of the Casimirs generates the ring of Hamiltonians which Poisson commutes for any of the structures. This can be thought of as an example of a generalization of the Gel'fand Zakharevich theorem [GZ]. In section 2 , we will give the three levels of phase space that we consider, for each of the three cases (rational, elliptic, trigonometric) that we are considering, and state the theorems that relate them. In section 3, we exhibit the multi-Hamiltonian structure, and the Nijenhuis coordinates. Section 4 is devoted to making some of the formulae explicit in the rational case, as well as some examples. 
We would like to thank Eyal Markman and Franco Magri for useful discussions.

\section{Phase spaces.}

A) The Lie-Poisson-Sklyanin phase spaces

i) Rational case

For this case, fixing an integer $n$, the phase space is simply

$$
\mathcal{M}_{\text {rat }}=\{g l(r, \mathbb{C})-\text { valued polynomials of degree } \leq n\}
$$

Let $\Sigma$ be $\mathbb{P}_{1}$, the Riemann sphere. Let $\mathcal{O}(m)$ denote the standard degree $m$ line bundle on $\Sigma$, and set $F(m)=F \otimes \mathcal{O}(m)$ for any vector bundle $F$. Let $K_{\Sigma}=\mathcal{O}(-2)$ be the canonical (=cotangent) bundle of $\Sigma$. If $E$ is the trivial rank $r$ vector bundle on $\Sigma, \mathcal{M}_{\text {rat }}$ can be reinterpreted as:

$$
\mathcal{M}_{\text {rat }}=H^{0}(\Sigma, E n d(E)(n))=H^{0}\left(\Sigma, E n d(E) \otimes K_{\Sigma}(n+2)\right),
$$

that is the global holomorphic sections of the endomorphisms of $E$ with a pole of order $n$ at infinity, or alternately, of the 1-form-valued endomorphisms of $E$ with a pole of degree $n+2$ at infinity.

The dual space to $\mathcal{M}_{\text {rat }}$, using the trace residue pairing $<a, b>=\operatorname{tr}\left(\operatorname{res}_{\infty}(a b)\right)$, can be identified with the space of matricial Laurent polynomials with entries of degree $-n-1, \ldots,-1$. For any pair $f, g$ of functions on $\mathcal{M}_{\text {rat }}$, therefore, the differentials $d f, d g$ at a point may be identified as such Laurent polynomials. In a more invariant fashion, these Laurent polynomials are representative cocycles for cohomology classes, and the dual space of $\mathcal{M}_{\text {rat }}$ is

$$
\mathcal{M}_{\text {rat }}^{*}=H^{1}(\Sigma, E n d(E)(-n-2)),
$$

This is just Serre duality. Let $P_{+}$be the projection defined on the space of all Laurent polynomials which is the identity on the terms of degree greater or equal to zero, and sets to zero the terms of strictly negative degree. Let $P_{-}$be the complementary projection: $P_{+}+P_{-}=\mathbb{I}$. We set

$$
R=P_{+}-P_{-} .
$$

On the space $\mathcal{M}_{\text {rat }}$, one has an $(n+3)$ dimensional family of Poisson structures defined as follows: let $a=a(\lambda)$ be a polynomial of degree at most $n+1$, and $b$ be a constant. Our Poisson structures at $\phi \in \mathcal{M}_{\text {rat }}$ are given by:

$$
\{f, g\}(\phi)=<\phi,[R(a d f), d g]+[d f, R(a d g)]>-\frac{b}{2}\left(<R(D f), D g>+<D^{\prime} f, R\left(D^{\prime} g\right)>\right)
$$

where $D$ denotes the left derivative and $D^{\prime}$ the right derivative. These are defined on tangent vectors $\dot{\phi}$ at $\phi$ by

$$
<D f, \dot{\phi} \phi^{-1}>=<d f, \dot{\phi}>=<D^{\prime} f, \phi^{-1} \dot{\phi}>
$$


so that $D f=\phi d f, D^{\prime} f=d f \phi$.

One can write the Poisson bracket in a different fashion. Noting that the Poisson brackets are determined by their values on the matrix entries $\phi_{i, j}(\lambda)$ as $i, j, \lambda$ vary, and that the projection $P_{+}(f)(\lambda)$ of a function $f$ can be defined by the contour integral

$$
P_{+}(f)(\lambda)=\frac{1}{2 \pi i} \oint \frac{1}{\mu-\lambda} f(\mu) d \mu
$$

we can obtain, after some computations:

$$
\{\phi(\lambda) \stackrel{\otimes}{,} \phi(\mu)\}_{a, b}:=\left[r(\lambda-\mu), \phi(\lambda) \otimes\left(a(\mu) \mathbb{I}-\frac{b}{2} \phi(\mu)\right)+\left(a(\lambda) \mathbb{I}-\frac{b}{2} \phi(\lambda)\right) \otimes \phi(\mu)\right],
$$

Here we use the tensor-bracket notation of $[\mathrm{FT}]$, considering both sides as elements of $\operatorname{End}\left(\mathbb{C}^{r} \otimes \mathbb{C}^{r}\right) ; r(\lambda-\mu)$ is the explicit expression of the $r$-matrix. When $b=0$, the Poisson structure is equivalent to the standard linear $r$-matrix bracket on the family of matrices of the form $a(\lambda)^{-1} \phi, \phi \in \mathcal{M}_{\text {rat }}$; when $a=0, b=-1$, the bracket is the standard quadratic (Sklyanin) bracket.

ii) Elliptic case: (cf. [FT])

Let $\Sigma$ be an elliptic curve, defined as

$$
\Sigma=\mathbb{C} /\left(\omega_{1} \mathbb{Z}+\omega_{2} \mathbb{Z}\right)
$$

and let $\pi: \mathbb{C} \rightarrow \Sigma$ be the natural projection.

Let $q=\exp (2 \pi i / r)$, and set

$$
I_{1}=\operatorname{diag}\left(1, q, q^{2}, \ldots, q^{r-1}\right), \quad I_{2}=\left(\begin{array}{ccccc}
0 & 1 & 0 & \ldots & 0 \\
0 & 0 & 1 & \ldots & 0 \\
. & . & . & & . \\
. & . & . & & . \\
0 & 0 & 0 & \ldots & 1 \\
1 & 0 & 0 & \ldots & 0
\end{array}\right) .
$$

Note that $I_{1} I_{2} I_{1}^{-1} I_{2}^{-1}=q^{-1} \mathbb{I}$.

Let $D$ be a divisor $\nu_{1}+\nu_{2}+\ldots+\nu_{n}$ on $\Sigma$, so that the $\nu_{i}$ are points (possibly repeated) on $\Sigma$.

Our phase space $\mathcal{M}_{\text {ell }}$ will be the product of the curve with the space of meromorphic functions on $\mathbb{C}$ with values in $g l(r, \mathbb{C})$, with poles only at the translates of the $\nu_{i}$ and satisfying the quasiperiodicity relations:

$$
\begin{aligned}
& \mathcal{M}_{\text {ell }}=\Sigma \times\{\phi \text { meromorphic } g l(r, \mathbb{C})-\text { valued functions on } \mathbb{C} \text { such that } \\
& \left.\qquad \phi^{-1}(\infty) \subset \cup_{i}\left\{\nu_{i}+\mathbb{Z} \omega_{1}+\mathbb{Z} \omega_{2}\right\} \text { and } \phi\left(\lambda+\omega_{i}\right)=I_{i} \phi(\lambda) I_{i}^{-1}, i=1,2 .\right\}
\end{aligned}
$$


The second factor can again be thought of as a space of meromorphic sections of the endomorphism bundle of a vector bundle, this time of degree 1 . The first factor is to be thought of as classifing the bundle. Indeed, referring to Atiyah [At], stable vector bundles $E$ of degree 1, rank $r$, are classified by their top exterior power $\Lambda^{r}(E) \in \operatorname{Pic}^{1}(\Sigma)=\Sigma$, and are all related to each other by tensoring by a line bundle: $E^{\prime}=E \times L$, so that in particular $E n d\left(E^{\prime}\right)=E n d(E)$. They all have a one-dimensional space of sections. By the theorem of Narasimhan and Seshadri[NS], stable bundles correspond to irreducible representations of a $\mathbb{Z}$-central extension of the fundamental group, in our case given by the matrices $\left(\tilde{I}_{1}, \tilde{I}_{2}\right)=\left(I_{1}, c I_{2}\right)$, for a constant $c$. Sections of the corresponding vector bundles $E_{c}$ correspond to vector valued functions on $\mathbb{C}$ satisfying the quasi-periodicity relations $\psi\left(\lambda+\omega_{i}\right)=\tilde{I}_{i} \psi(\lambda)$. Sections of $E n d\left(E_{c}\right)$ in turn, are given by holomorphic matrix valued functions on $\mathbb{C}$, satisfying the quasi-periodicity relations of (2.11) on $\mathbb{C}$. Setting $E=E_{1}$, the space $\mathcal{M}_{\text {ell }}$ then gets interpreted as sections of $E n d(E)$, meromorphic, with poles at $D$ :

$$
\mathcal{M}_{\text {ell }}=\Sigma \times H^{0}(\Sigma, E n d(E)(D))=\Sigma \times H^{0}\left(\Sigma, E n d(E) \otimes K_{\Sigma}(D)\right) .
$$

Corresponding to the fact that the vector bundle $E$ is rigid once one fixes the top exterior power, one has $H^{1}(\Sigma, \operatorname{sl}(E))=0$, and dually, $H^{0}(\Sigma, \operatorname{sl}(E))=0$. This, in concrete terms, means that any section $s$ of $s l(E)$ on a punctured neighbourhood of the origin in $\Sigma$ decomposes uniquely into $s_{+}+s_{-}$, where $s_{+}$is a section defined on an unpunctured neighbourhood $U_{+}$of the origin, and $s_{-}$is defined on $U_{-}=\Sigma-$ \{origin $\}$. Set $P_{+}(s)=s_{+}, P_{-}(s)=s_{-}$. Using the representation of sections of $E$ given above, another way of giving this decomposition is to say that any meromorphic $\operatorname{sl}(r, \mathbb{C})$-valued function $f$ on a punctured neighbourhood of the origin in $\Sigma$ decomposes uniquely into $f_{+}+f_{-}$, where $f_{+}$is defined on an unpunctured neighbourhood of the origin in $\mathbb{C}$, and $f_{-}$is defined on $\mathbb{C}-\{$ translates of the origin $\}$, and satisfies the quasi-periodicity relations of (2.11). Again, we denote the corresponding projections by $P_{+}, P_{-}$.

More generally, a section $\rho$ of $\operatorname{End}(E)$ on a punctured neighbourhood of the origin in $\Sigma$ can be decomposed into its trace component $P_{0}(\rho)$ and its $s l(r, \mathbb{C})$ component $\rho-P_{0}(\rho)$; in turn, the latter decomposes into its components $P_{+}(\rho)=P_{+}\left(\rho-P_{0}(\rho)\right), P_{-}(\rho)=$ $P_{-}\left(\rho-P_{0}(\rho)\right)$, so that $\rho=P_{+}(\rho)+P_{-}(\rho)+P_{0}(\rho)$. As before, set $R=P_{+}-P_{-}$.

The cotangent space of $\mathcal{M}_{\text {ell }}$ at $\left(E_{c}, \phi\right)$ is identified with $\mathbb{C} \times H^{1}(\Sigma, E n d(E)(-D))$ : the differential $d f$ of a function $f$ on $\mathcal{M}_{\text {ell }}$ splits into $d f_{E} \in \mathbb{C}, d f_{\phi} \in H^{1}(\Sigma, E n d(E)(-D))$. The line bundle $\mathcal{O}(D)$ on $\Sigma$, whose sections correspond to meromorphic functions with poles only at $D$, has an $n$-dimensional space $V$ of global sections. If $a \in V$, representing $d f_{\phi}$ by a cocycle one has that $a d f_{\phi}$ is a cocycle with values in $E n d(E)$, and so can be split into its,,+- 0 components; similarly, $D f_{\phi}=\phi d f_{\phi}, D^{\prime} f_{\phi}=d f_{\phi} \phi$ also lie in $E n d(E)$, and can also be split.

One can define an $n+1$-dimensional family of Poisson structures on $\mathcal{M}_{\text {ell }}$ by

$$
\begin{aligned}
\{f, g\}(\phi)= & <\phi,\left[R\left(a d f_{\phi}\right), d g_{\phi}\right]+\left[d f_{\phi}, R\left(a d g_{\phi}\right)\right]> \\
& -\frac{b}{2}\left(<R\left(D f_{\phi}\right), D g_{\phi}>+<D^{\prime} f_{\phi}, R\left(D^{\prime} g_{\phi}\right)>\right) \\
& +<P_{0}\left(a d f_{\phi}+b D f_{\phi}\right), d g_{E}>-<d f_{E}, P_{0}\left(\left(a d g_{\phi}+b D g_{\phi}\right)>,\right.
\end{aligned}
$$


where $a$ lies in $V$, and $b$ is a constant. The case $b=0$ again corresponds to the linear $r$-matrix bracket; the case $a=0$ gives the quadratic bracket. One can again write out a formula as in (2.8) for the bracket, this time involving elliptic functions.

\section{Reduction to $\operatorname{sl}(r)$}

The more familiar form of either the linear or quadratic elliptic $r$-matrix bracket lives on $\operatorname{sl}(r, \mathbb{C})$-valued functions, in the linear case, or on $\operatorname{Sl}(r, \mathbb{C})$-valued functions, in the quadratic case. In our context, these arise by first fixing the values of some Casimirs, then reducing. The projection to the first factor in $\mathcal{M}_{\text {ell }}$ gives a Hamiltonian function, which acts on the pairs $(c, \phi)$ by

$$
(\dot{c}, \dot{\phi})=(0, a+b \phi) .
$$

Reducing then amounts to fixing the first factor in $\mathcal{M}_{\text {ell }}$ (i.e., fixing the bundle), and then quotienting by the flow (2.14).

When $b=0$, the flow acts by adding to $\phi$ a multiple of the identity. The polar parts of the trace components are Casimirs; we set these to zero. The easy normalisation for quotienting by the flow is given by fixing the constant term of the trace, and so we set this to zero also, so that the whole trace term vanishes. We are now in $\operatorname{sl}(r, \mathbb{C})$, with a reduced phase space

$$
\begin{aligned}
& \left(\mathcal{M}_{\text {ell }}\right)_{\text {red }}=\{\phi \text { meromorphic } \operatorname{sl}(r, \mathbb{C})-\text { valued functions on } \mathbb{C} \text { such that } \\
& \left.\qquad \phi^{-1}(\infty) \subset \cup_{i}\left\{\nu_{i}+\mathbb{Z} \omega_{1}+\mathbb{Z} \omega_{2}\right\} \text { and } \phi\left(z+\omega_{i}\right)=I_{i} \phi(z) I_{i}^{-1}, i=1,2\right\} .
\end{aligned}
$$

Similarily, when $a=0$, the zeroes and poles of the determinant are Casimirs, and the flow rescales the section $\phi$, so that choosing leaves for which the determinant is constant, and then rescaling so that the determinant along the symplectic leaves is 1 lands us in $S l(r, \mathbb{C})$, giving us a space which is the reduction of the previous one up to a finite ambiguity:

$$
\begin{aligned}
& \left(\mathcal{M}_{\text {ell }}\right)_{\text {red }}=\{\phi \text { meromorphic } S l(r, \mathbb{C})-\text { valued functions on } \mathbb{C} \text { such that } \\
& \left.\qquad \phi^{-1}(\infty) \subset \cup_{i}\left\{\nu_{i}+\mathbb{Z} \omega_{1}+\mathbb{Z} \omega_{2}\right\} \text { and } \phi\left(\lambda+\omega_{i}\right)=I_{i} \phi(\lambda) I_{i}^{-1}, i=1,2\right\} .
\end{aligned}
$$

\section{iii) Trigonometric case}

This case can be thought of as a degeneration of the elliptic case. The elliptic curve degenerates into a Riemann sphere $\Sigma=\mathbb{P}_{1}$ with two points $z=0, \infty$ identified. The smooth part of the curve is then $\mathbb{C}^{*}$. Let $\pi: \mathbb{C} \rightarrow \mathbb{C}^{*}$ be the map $z \mapsto \exp (2 \pi i z)$.

Let $D$ represent a sum $\nu_{1}+\nu_{2}+\ldots+\nu_{n}$ of points $\nu_{i}$ on $\mathbb{C}^{*}$.

Our phase space $\mathcal{M}_{\text {trig }}$ will be the product of $\mathbb{C}^{*}$ with the space of $\operatorname{sl}(r, \mathbb{C})$-valued meromorphic functions $\phi$ on $\mathbb{C}$, with poles at $D$, satisfying

$$
\begin{gathered}
\phi(\lambda+1)=I_{1} \phi(\lambda) I_{1}^{-1} \\
\lim _{\operatorname{Re}(-i \lambda) \rightarrow+\infty} \phi=\lim _{\operatorname{Re}(-i \lambda) \rightarrow-\infty} I_{2} \phi(\lambda) I_{2}^{-1} .
\end{gathered}
$$


As for the elliptic case, $\mathcal{M}_{\text {trig }}$ can be expressed as the space

$$
\mathcal{M}_{\text {trig }}=\mathbb{C}^{*} \times H^{0}\left(\Sigma, E n d(E) \otimes K_{\Sigma}(D)\right),
$$

for a suitable stable degree one vector bundle $E$ on $\Sigma$. The bundle is obtained from the sum of line bundles $\mathcal{O} \oplus \mathcal{O} \oplus \ldots \oplus \mathcal{O} \oplus \mathcal{O}(1)$ on $\mathbb{P}_{1}$ by identifying the fibers over $0, \infty$ in the standard trivialisations by the matrix:

$$
A=\left(\begin{array}{cccccc}
0 & 0 & 0 & \ldots & 0 & 1 \\
1 & 0 & 0 & \ldots & 0 & 0 \\
\cdot & . & . & & . & \\
\cdot & \cdot & \cdot & & \cdot & \\
& & & & & \\
0 & 0 & 0 & \ldots & 1 & 0
\end{array}\right)
$$

As in the elliptic case, the extra $\mathbb{C}^{*}$ factor represents twists of $E$ by a line bundle; this translates here into a rescaling of the matrix $A$ by a scalar factor, and does not change the explicit expression of the endomorphisms. Exactly as in the elliptic case, one has $H^{1}(\Sigma, s l(E))=H^{0}(\Sigma, s l(E))=0$, and so projections $P_{+}, P_{-}$and their difference $R$, as well as a projection $P_{0}$ onto the trace component. Again, the line bundle $\mathcal{O}(D)$ has an $n$-dimensional space of sections, and there is an $n+1$-dimensional family of Poisson structures on $\mathcal{M}_{\text {trig }}$ defined by the formula (2.13).

\section{B) Spectral curves and line Bundles.}

The three moduli spaces given above are particular examples of moduli spaces of Higgs pairs. For any compact Riemann surface $\Sigma$, and positive divisor $D$ of degree $n$ on $\Sigma$, one can consider [Ma], [Bo] the moduli space $\mathcal{H}(r, D, d)$ of Higgs pairs $(E, \phi)$, where

- $E$ is a degree $d$ rank $r$ holomorphic vector bundle over $\Sigma$.

- $\phi$, the Higgs field, is a holomorphic section of the associated adjoint bundle $\operatorname{End}(E)$, twisted by $K_{\Sigma}(D)$, where $K_{\Sigma}$ is the canonical bundle of $\Sigma: \phi \in H^{0}(\Sigma, \operatorname{End}(E) \otimes$ $\left.K_{\Sigma}(D)\right)$. Alternately, $\phi$ is a meromorphic $\operatorname{End}(E)$-valued 1-form, with poles at the divisor $D$.

To each pair $(E, \phi)$, one can associate the spectral curve $S$ of $\phi$. This curve lies in the total space $T$ of the line bundle $K(D)$ over $\Sigma$. It is cut out by the equation

$$
\operatorname{det}(\phi(\lambda)-\zeta \mathbb{I})=0 .
$$

Here $\zeta$ represents the tautological section of $\pi^{*} K(D)$ over $T$, where $\pi: T \rightarrow \Sigma$ is the projection. The projection $\pi$ exhibits $S$ as an $r$-sheeted branched cover of $\Sigma$. If $\gamma$ is the genus of $\Sigma$, the genus of $S$ is

$$
g=r^{2}(\gamma-1)+\frac{(r-1) r n}{2}+1 .
$$


One can also define a sheaf $L$ supported on $\Sigma$, by the exact sequence over the surface $\mathcal{K}_{D}$ :

$$
0 \rightarrow \pi^{*} E \otimes K_{\Sigma}^{*}(-D) \stackrel{\phi-\zeta \mathbb{I}}{\longrightarrow} \pi^{*} E \rightarrow L \rightarrow 0
$$

On the generic locus for which $S$ is smooth and the eigenspaces are line bundles, $L$ will be a line bundle over $S$, of degree $d+r(1-\gamma)+g-1$.

One has:

Proposition (2.24) [Hu] One can reconstruct $(E, \phi)$ from $(S, L)$ :

- $E=\pi_{*}(L)$,

- $\phi$ is, up to automorphisms, the map induced on $E$ by multiplication by the tautological section $\zeta$ on $L$.

Our spaces $\mathcal{M}$ are all dense open sets in the moduli $\mathcal{H}(r, D, d)$; the preceeding theorem will allow us to describe the quotient $\mathcal{N}=\mathcal{M} / A$ ut of $\mathcal{H}(r, D, d)$ by a (constant over $\mathcal{M}$ ) group of automorphisms, as a space of pairs $(S, L)$; alternately, rather than consider pairs $(S, L)$, we note that when one thinks of $L$ as a sheaf over the surface $\mathcal{K}_{D}$, the curve $S$ is the support of $L$, and so is specified by it. The space $\mathcal{N}$ is then isomorphic to a space of sheaves $L$ supported on curves. The tangent space at $L$ is then given by the global Ext-group

$$
T \mathcal{N}_{L}=\operatorname{Ext}^{1}(L, L)
$$

and the cotangent space by

$$
T^{*} \mathcal{N}_{L}=\operatorname{Ext}^{1}\left(L, L \otimes K_{T}\right)
$$

whose computations are explained below. A Poisson structure is then given by a map

$$
\Lambda: \operatorname{Ext}^{1}\left(L, L \otimes K_{T}\right) \rightarrow \operatorname{Ext}^{1}(L, L) .
$$

It is the fundamental observation of Mukai, Tyurin and Bottacin [Mu, Ty, Bo] that a Poisson structure $\theta \in H^{0}\left(T, K_{T}^{*}\right)$ on the surface $T$ allows one to define such a structure, via the map $L \otimes K_{T} \rightarrow L$ that it induces. We will see that the surfaces which we consider have large families of Poisson structures, and that they correspond to the families of Poisson brackets given above.

We consider each of our three cases.

i) Rational case.

Here we take bundles of degree zero. The generic $(E, \phi)$ in this case is such that $E$ is a trivial bundle; $\phi$ is then a matrix valued function of degree $n$ with poles at $D$. One can multiply by a scalar polynomial, and take all poles to be at infinity, normalising to $D=n \infty$. One then has

$$
\mathcal{M}_{\text {rat }} \subset \mathcal{H}(r, D, 0),
$$

as the open subset for which $E$ is trivial. On this subset, the automorphisms of $E$ are then given by constant matrices in $G l(r, \mathbb{C})$; under the isomorphism above, they act on $\mathcal{M}_{\text {rat }}$ 
by conjugation, and if we let $\mathcal{N}_{\text {rat }}$ be the variety of pairs $(S, L)$ corresponding to elements of $\mathcal{M}_{\text {rat }}$, one has

$$
\mathcal{M}_{\text {rat }} / G l(r, \mathbb{C})=\mathcal{N}_{\text {rat }}
$$

By (2.22), the genus of the spectral curves is $g=-r^{2}+\frac{(r-1) r n}{2}+1$, and the degree of the line bundles $L$ when the curve is smooth is $g+r-1$. The spectral curve is embedded in the total space $T$ of the line bundle $\mathcal{O}(n)$ over $\mathbb{P}^{1}$. The anticanonical bundle of $T$ is the lift from $\mathbb{P}^{1}$ of $\mathcal{O}(n+2)$. Poisson structures are then sections of this bundle: the space of Poisson structures on $T$ is then of dimension $n+6$. If $\lambda$ is the standard coordinate on $\mathbb{P}^{1}$, and $z$ is a standard fiber coordinate corresponding to the tautological section $\zeta$ (so that $T$ is covered by two coordinate systems $(\lambda, z),(\tilde{\lambda}, \tilde{z})$ related by $\left.(\tilde{\lambda}, \tilde{z})=\left(\lambda^{-1}, z \lambda^{-n}\right)\right)$, the Poisson structures are given by

$$
(a(\lambda)+b(\lambda) z)\left(\frac{\partial}{\partial \lambda} \wedge \frac{\partial}{\partial z}\right)
$$

where $a$ is a polynomial of degree at most $(n+2)$ and $b$ a polynomial of degree at most 2 . We restrict to the $(n+3)$-dimensional space $W$ of polynomials $a, b$ of degrees at most $(n+1), 0$, respectively; these Poisson structures on the surface vanish over $\lambda=\infty$.

One has $[\mathrm{AHH}],[\mathrm{Sc}],[\mathrm{HuMa}]$ :

Proposition (2.30) The Mukai structures that the Poisson structures in $W$ induce on the space $\mathcal{M}_{\text {rat }} / G l(n, \mathbb{C})$ are the same as the reductions of the Poisson structures given in section a).

As the references given are rather scattered and in some respects only partial, we give here a sketch of the proof, generalising [HuMa]. The Mukai Poisson structure is given by a map from the cotangent space to the tangent space

$$
\Lambda: \operatorname{Ext}^{1}\left(L, L \otimes K_{\mathcal{T}}\right) \rightarrow \operatorname{Ext}^{1}(L, L) . \operatorname{eqno}(2.31)
$$

To compute the Ext-groups, one must first take a resolution $R$ of $L$, take the induced sequence $\operatorname{Hom}(R, L)$, and then compute the first hypercohomology group of this sequence, which we will do explicitly below. We choose the resolution of (2.23). Applying Hom, and recalling that $K_{\mathcal{T}}=\pi^{*} \mathcal{O}(-n-2)$, the cotangent space will be the first hypercohomology of the complex $C_{T^{*}}$ supported over the spectral curve

$$
\left(\pi^{*} E\right)^{*} \otimes L \otimes \pi^{*} \mathcal{O}(-n-2) \stackrel{(\phi-\zeta \mathbb{I})^{*}}{\longrightarrow}\left(\pi^{*} E\right)^{*} \otimes L \otimes \pi^{*} \mathcal{O}(-2),
$$

and the tangent space the first hypercohomology of the complex $C_{T}$

$$
\left(\pi^{*} E\right)^{*} \otimes L \stackrel{(\phi-\zeta \mathbb{I})^{*}}{\longrightarrow}\left(\pi^{*} E\right)^{*} \otimes L \otimes \pi^{*} \mathcal{O}(n) .
$$

This is supported on the spectral curve. The map from the cotangent space to the tangent space is induced by termwise multiplication in the resolution by the Poisson structure $(a(\lambda)+b(\lambda) z)\left(\frac{\partial}{\partial \lambda} \wedge \frac{\partial}{\partial z}\right)$. Following $[\mathrm{Ma}]$, one can push down to $\mathbb{P}_{1}$, to have a diagram

$$
\begin{aligned}
& \operatorname{End}(E)(-n-2) \quad \stackrel{a d_{\phi}}{\longrightarrow} \quad \operatorname{End}(E)(-2) \\
& \downarrow(a+b \phi) . \quad \downarrow(a+b \phi) . \\
& \operatorname{End}(E) \quad \stackrel{a d_{\phi}}{\longrightarrow} \quad \operatorname{End}(E)(n) .
\end{aligned}
$$


The first hypercohomology of the top row is the cotangent space of $\mathcal{N}_{\text {rat }}$; that of the bottom row is the tangent space; the vertical maps induce the Poisson structure.

The first hypercohomology $\mathbb{H}^{1}$ of a sequence of sheaves $R \stackrel{\rho}{\longrightarrow} S$ is given in terms of Cech cocycles (defined with respect to the standard cover $U_{+}, U_{-}$of $\mathbb{P}_{1}$ ) by equivalence classes of pairs $\left(r_{ \pm}, s_{+}, s_{-}\right)$where $r_{ \pm}$is a section of $R$ over $U_{+} \cap U_{-}, s_{+}, s_{-}$are sections of $S$ over $U_{+}, U_{-}$respectively (a 0 -cochain), and $r, s$ satisfy $\rho\left(r_{ \pm}\right)-s_{+}+s_{-}=0$ on $U_{+} \cap U_{-}$. The equivalence relation is that one can modify $\left(r_{ \pm}, s_{+}, s_{-}\right)$by a coboundary $\left(\hat{r}_{+}-\hat{r}_{-}, \rho\left(\hat{r}_{+}\right), \rho\left(\hat{r}_{-}\right)\right)$for 0 -cochains $\hat{r}$ with values in $R$. In particular, one sees that the hypercohomology group $\mathbb{H}^{1}$ maps to $H^{1}(R)$ with $H^{0}(S) / \rho\left(H^{0}(R)\right)$ in the kernel.

In our case, as the bundle is trivial, we have $H^{1}\left(\mathbb{P}_{1}, E n d(E)\right)=0$ : the bundle is rigid. In particular, we have complementary projections $P_{+}, P_{-}$from $H^{0}\left(U_{+} \cap U_{-}, E n d(E)\right)$ to $H^{0}\left(U_{+}, E n d(E)\right), H^{0}\left(U_{-}, E n d(E)\right)$ respectively, which coincide with the projections defined above. Also, $H^{0}\left(\mathbb{P}_{1}, E n d(E)\right)=g l(r, \mathbb{C})$. If $C_{T}$ is the tangent complex in (2.34), this gives the isomorphism

$$
H^{0}\left(\mathbb{P}_{1}, \operatorname{End}(E)(n)\right) /[\phi, g l(r, \mathbb{C})] \rightarrow \mathbb{H}^{1}\left(C_{T}\right)=T \mathcal{N}_{\text {rat }},
$$

Dually, we have $H^{0}\left(\mathbb{P}_{1}, E n d(E)(-2)\right)=0, H^{1}\left(\mathbb{P}_{1}, E n d(E)(-2)\right)=g l(r, \mathbb{C})^{*} ;$ furthermore, again using Serre duality, the dual space to $H^{0}\left(\mathbb{P}_{1}, E n d(E)(n)\right)$ is $H^{1}\left(\mathbb{P}_{1}, E n d(E)(-n-2)\right)$. The phase space $H^{0}\left(\mathbb{P}_{1}, E n d(E)(n)\right)$ is identified with the space of matricial polynomials of degree at most $n$, and dually, we represent elements of elements of $H^{1}\left(\mathbb{P}_{1}, E n d(E)(-n-2)\right)$ by matrix valued Laurent polynomials with terms of degree $-n-1$ to -1 ; the Serre duality pairing is then trace-residue. Thus, if $C_{T^{*}}$ denotes the cotangent complex, there is an isomorphism

$$
\mathbb{H}^{1}\left(C_{T^{*}}\right)=T^{*} \mathcal{N}_{\text {rat }} \rightarrow\left(k e r[\phi, \cdot]: H^{1}\left(\mathbb{P}_{1}, \operatorname{End}(E)(-n-2)\right) \rightarrow H^{1}\left(\mathbb{P}_{1}, \operatorname{End}(E)(-2)\right)\right) .
$$

Let us compute the Poisson tensor $\Lambda$. An element of the cotangent space $T^{*} \mathcal{N}_{\text {rat }}$ represented by a cocycle $c_{ \pm}$lifts to a hypercohomology cocycle $\left(c_{ \pm}, d_{+}, d_{-}\right)$with values in $\operatorname{End}(E)(-n-2), \operatorname{End}(E)(-2)$ respectively, satisfying $\left[\phi, c_{ \pm}\right]=d_{+}-d-$, so that one can take $d_{+}=P_{+}\left(\left[\phi, c_{ \pm}\right]\right), d_{-}=-P_{-}\left(\left[\phi, c_{ \pm}\right]\right)$. The Poisson tensor $\Lambda$ acts by

$$
\Lambda:\left(c_{ \pm}, d_{+}, d_{-}\right) \mapsto(a(\lambda)+b(\lambda) \phi)\left(c_{ \pm}, d_{+}, d_{-}\right) .
$$

We can modify the expression by a coboundary $\frac{-b}{2}\left(\left[\phi, c_{ \pm}\right],\left[\phi, d_{+}\right],\left[\phi, d_{-}\right]\right)$so that the eventual expression for the Poisson tensor will be more explicitly skew-symmetric:

$$
\Lambda:\left(c_{ \pm}, d_{+}, d_{-}\right) \mapsto\left(\left(a(\lambda)+\frac{b(\lambda)}{2} \phi\right)\left(c_{ \pm}, d_{+}, d_{-}\right)+\frac{b(\lambda)}{2}\left(c_{ \pm}, d_{+}, d_{-}\right) \phi\right) .
$$

As $H^{1}\left(\mathbb{P}_{1}, E n d(E)\right)=0$, we can split $a c_{ \pm}+\frac{b}{2}\left(\phi c_{ \pm}+c_{ \pm} \phi\right)$ using $P_{+}, P_{-}$, and modify our cocycle by the coboundary of $P_{ \pm}\left(a c_{ \pm}+\frac{b}{2}\left(\phi c_{ \pm}+c_{ \pm} \phi\right)\right)$. This gives the equivalent hypercohomology cocycle

$$
\begin{aligned}
(0, & a P_{+}\left(\left[\phi, c_{ \pm}\right]\right)+\frac{b}{2}\left(\phi P_{+}\left(\left[\phi, c_{ \pm}\right]\right)+P_{+}\left(\left[\phi, c_{ \pm}\right]\right) \phi\right)-\left[\phi, P_{+}\left(a c_{ \pm}+\frac{b}{2}\left(\phi c_{ \pm}+c_{ \pm} \phi\right)\right)\right], \\
- & \left.a P_{-}\left(\left[\phi, c_{ \pm}\right]\right)-\frac{b}{2}\left(\phi P_{-}\left(\left[\phi, c_{ \pm}\right]\right)+P_{-}\left(\left[\phi, c_{ \pm}\right]\right) \phi\right)+\left[\phi, P_{-}\left(a c_{ \pm}+\frac{b}{2}\left(\phi c_{ \pm}+c_{ \pm} \phi\right)\right)\right]\right),
\end{aligned}
$$


landing in the subspace $H^{0}\left(\mathbb{P}_{1}, \operatorname{End}(E)(n)\right)$, giving:

$$
\begin{aligned}
\Lambda: & H^{1}\left(\mathbb{P}_{1}, \operatorname{End}(E)(-n-2)\right) \rightarrow H^{0}\left(\mathbb{P}_{1}, \operatorname{End}(E)(n)\right) \\
& c_{ \pm} \mapsto a P_{+}\left(\left[\phi, c_{ \pm}\right]\right)+\frac{b}{2}\left(\phi P_{+}\left(\left[\phi, c_{ \pm}\right]\right)+P_{+}\left(\left[\phi, c_{ \pm}\right]\right) \phi\right)-\left[\phi, P_{+}\left(a c_{ \pm}+\frac{b}{2}\left(\phi c_{ \pm}+c_{ \pm} \phi\right)\right)\right] \\
& =-a P_{-}\left(\left[\phi, c_{ \pm}\right]\right)-\frac{b}{2}\left(\phi P_{-}\left(\left[\phi, c_{ \pm}\right]\right)+P_{-}\left(\left[\phi, c_{ \pm}\right]\right) \phi\right)+\left[\phi, P_{-}\left(a c_{ \pm}+\frac{b}{2}\left(\phi c_{ \pm}+c_{ \pm} \phi\right)\right)\right] .
\end{aligned}
$$

Let us compute the Poisson bracket corresponding to this, on a pair of functions $f, g$ on $H^{0}\left(\mathbb{P}_{1}, \operatorname{End}(E)(n)\right)$. The differentials $d f, d g$ of these functions at $\phi$ are naturally identified with classes in $H^{1}\left(\mathbb{P}_{1}, E n d(E)(-n-2)\right)$. Recall that $D f=\phi \cdot d f$, and $D^{\prime} f=d f \cdot \phi$, and similarly for $d g$. We have

$$
\begin{aligned}
\{f, g\}(\phi)= & <d f, \Lambda(d g)> \\
= & <d f, a P_{+}([\phi, d g])-\left[\phi, P_{+}(a d g)\right]> \\
& +<\frac{b}{2}\left(D f+D^{\prime} f\right), P_{+}\left(D g-D^{\prime} g\right)>-<D^{\prime} f-D f, P_{+}\left(\frac{b}{2}\left(D g+D^{\prime} g\right)\right)>.
\end{aligned}
$$

Using the identities

$$
\begin{aligned}
<P_{ \pm}(f), g> & =<f, P_{\mp}(g)> \\
<D f, D g> & =<D^{\prime} f, D^{\prime} g>
\end{aligned}
$$

we obtain:

$$
\begin{aligned}
\{f, g\}(\phi)= & <\phi,[R(a d f), d g]+[d f, R(a d g)]> \\
& +\frac{1}{2}\left(<R\left(b\left(D f+D^{\prime} f\right)\right), D^{\prime} g-D g>+<D f-D^{\prime} f, R\left(b\left(D g+D^{\prime} g\right)\right)>\right.
\end{aligned}
$$

which, when $b$ is a constant, reduces to our brackets $(2.5)$.

REMARK: One might hope that the formula (2.5) would define a Poisson bracket for the structures not in $W$. Unfortunately, for cases like $a=0, b=\lambda$, the Jacobi identity is not satisfied on the unreduced space. There does not seem to be any natural way of modifying the formula (2.5) (i.e. its lift to the unreduced space) so that the Jacobi identity is satisfied.

ii) The elliptic case.

The bundles $E^{\prime}$ we consider over our elliptic curve are of degree one; on the open set $\mathcal{M}_{\text {ell }}$ of $\mathcal{H}(r, D, 1)$ corresponding to the stable bundles, we have that $E^{\prime}=E \otimes L^{\prime}$, where $L^{\prime}$ is a line bundle of degree zero and $E$ is the vector bundle defined above. Any two such bundles $E^{\prime}, E^{\prime \prime}$ are isomorphic iff the corresponding line bundles $L^{\prime}, L^{\prime \prime}$ are such that $\left(L^{\prime} \otimes\left(L^{\prime \prime}\right)^{*}\right)^{\otimes r}$ is trivial, that is if $\left(L^{\prime} \otimes\left(L^{\prime \prime}\right)^{*}\right.$ is an $r$-th root of unity in $\operatorname{Pic}^{0}(\Sigma) \simeq \Sigma[\mathrm{At}]$, so that the moduli space of bundles is then the torus $\Sigma \simeq \operatorname{Pic}(\Sigma) /(\mathbb{Z} / r)^{2}$. The group Aut of global automorphisms of the stable bundles are constant multiples of the identity, so that $\mathcal{N}_{\text {ell }}=\mathcal{M}_{\text {ell }} /$ Aut $=\mathcal{M}_{\text {ell }}$. 
By (2.22), the genus of the spectral curves is $g=\frac{(r-1) r n}{2}+1$, and the degree of the line bundles $L$ when the curve is smooth is $g$. The spectral curve is embedded in the total space $T$ of the line bundle $\mathcal{O}(D)$ over $\Sigma$. The anticanonical bundle of $T$ is the lift from $\Sigma$ to $T$ of $\mathcal{O}(D)$. Poisson structures are then sections of this bundle: the space of Poisson structures on $T$ is of dimension $n+1$; the Poisson structures are of the form

$$
(a+b z)\left(\frac{\partial}{\partial \lambda} \wedge \frac{\partial}{\partial z}\right),
$$

where $a$ is a section of $\mathcal{O}(D)$ lifted from $\Sigma, b \in \mathbb{C}$ and $z$ is the tautological section of $\pi^{*}(\mathcal{O}(D))$ on $T$. We then have an $(n+1)$ - dimensional family of Mukai brackets on $\mathcal{N}_{\text {ell }}$.

Proposition (2.45) [HuK, HuMa] We have:

$$
\mathcal{M}_{\text {ell }}=\mathcal{N}_{\text {ell }} .
$$

The Mukai Poisson structures on $\mathcal{N}_{\text {ell }}$ are equivalent those on $\mathcal{M}_{\text {ell }}$ given above.

Proof: The identification of the spaces is given above; what remains to be done is to identify the Poisson tensors. As for the rational case, we have a diagram:

$$
\begin{array}{ccc}
\operatorname{End}(E)(-D) & \stackrel{a d_{\phi}}{\longrightarrow} & \operatorname{End}(E) \\
\downarrow(a+b \phi) . & & \downarrow(a+b \phi) . \\
\operatorname{End}(E) & \stackrel{a d_{\phi}}{\longrightarrow} & \operatorname{End}(E)(D) .
\end{array}
$$

The first hypercohomology of the bottom row is the tangent space of $\mathcal{N}_{\text {ell }}$; it decomposes as a sum

$$
H^{1}(\Sigma, \mathcal{O}) \oplus H^{0}(\Sigma, \operatorname{End}(E)(D)) .
$$

The first factor corresponds to the tangent space of $\Sigma$ in $\mathcal{M}_{\text {ell }}$. Dually, the cotangent space is a sum

$$
H^{1}(\Sigma, \operatorname{End}(E)(-D)) \oplus H^{0}(\Sigma, \mathcal{O}) .
$$

We recall from above that since the bundles are rigid up to tensoring by a line bundle, we have $H^{1}(\Sigma, s l(E))=0$, and so we have complementary projections $P_{+}, P_{-}, P_{0}$ from $H^{0}\left(U_{+} \cap U_{-}, \operatorname{End}(E)\right)$ to $H^{0}\left(U_{+}, s l(E)\right), H^{0}\left(U_{-}, s l(E)\right), H^{0}\left(U_{+} \cap U_{-}, \mathcal{O}\right)$ respectively.

To compute the Poisson tensor, we take a cocycle $c_{ \pm}$representing an element of $H^{1}(\Sigma, \operatorname{End}(E)(-D))$, and a constant $c_{E} \in H^{0}(\Sigma, \mathcal{O})$. This corresponds to a hypercohomology cocycle

$$
\left(c_{ \pm}, d_{+}+c_{E} \mathbb{I}, d_{-}+c_{E} \mathbb{I}\right)
$$

where $d_{+}=P_{+}\left(\left[\phi, c_{ \pm}\right]\right), d_{-}=-P_{-}\left(\left[\phi, c_{ \pm}\right]\right)$. This gets mapped by $\Lambda$ to $(a+b \phi)\left(c_{ \pm}, d_{+}+\right.$ $\left.c_{E} \mathbb{I}, d_{-}+c_{E} \mathbb{I}\right)$. We can split $(a+b \phi) c_{ \pm}$using $P_{+}, P_{-}, P_{0}$ and modify our cocycle by the coboundary of $\pm P_{ \pm}\left((a+b \phi) c_{ \pm}\right)$. This gives the equivalent cocycle

$$
\begin{aligned}
\left(P_{0}\left((a+b \phi) c_{ \pm}\right) \mathbb{I}, \quad(a+b \phi) P_{+}\left(\left[\phi, c_{ \pm}\right]\right)-\left[\phi, P_{+}\left((a+b \phi) c_{ \pm}\right)\right]+(a+b \phi) c_{E} \mathbb{I},\right. \\
\left.-(a+b \phi) P_{-}\left(\left[\phi, c_{ \pm}\right]\right)+\left[\phi, P_{-}\left((a+b \phi) c_{ \pm}\right)\right]+(a+b \phi) c_{E} \mathbb{I}\right)
\end{aligned}
$$


landing in the $H^{1}(\Sigma, \mathcal{O}) \oplus H^{0}(\Sigma, E n d(E)(D))$, giving:

$$
\begin{aligned}
\Lambda: H^{1}(\Sigma, \operatorname{End}(E)(-D)) \oplus H^{0}(\Sigma, \mathcal{O}) & \rightarrow H^{1}(\Sigma, \mathcal{O}) \oplus H^{0}(\Sigma, \operatorname{End}(E)(D)), \\
\left(c_{ \pm}, c_{E}\right) \quad \mapsto\left(P_{0}((a+b \phi) c \pm), \quad(a+b \phi) P_{+}\left(\left[\phi, c_{ \pm}\right]\right)\right. & \left.-\left[\phi, P_{+}\left((a+b \phi) c_{ \pm}\right)\right]+(a+b \phi) c_{E} \mathbb{I}\right) .
\end{aligned}
$$

Let us compute the Poisson bracket corresponding to this, on a pair of functions $f, g$ on $\mathcal{N}_{\text {ell }}$. The differentials $d f, d g$ of these functions at $\phi$ are naturally identified with classes $\left(d f_{\phi}, d f_{E}\right),\left(d g_{\phi}, d g_{E}\right) \in H^{1}(\Sigma, E n d(E)(-D)) \oplus H^{0}(\Sigma, \mathcal{O})$. Recall that $D f=\phi \cdot d f$, and $D^{\prime} f=d f \cdot \phi$, and similarly for $d g$. We have

$$
\begin{aligned}
\{f, g\}(\phi)= & <d f, \Lambda(d g)> \\
= & <d f_{\phi}, a P_{+}\left(\left[\phi, d g_{\phi}\right]\right)-\left[\phi, P_{+}\left(a d g_{\phi}\right)\right]> \\
& -b<D^{\prime} f_{\phi}, P_{+}\left(D^{\prime} g_{\phi}\right)>+b<D f_{\phi}, P_{+}\left(D g_{\phi}\right)> \\
& +<\left(d f_{\phi}\right),(a+b \phi) d g_{E} \mathbb{I}> \\
& -<d f_{E}, P_{0}\left((a+b \phi) d g_{\phi}\right)>.
\end{aligned}
$$

Using the identities (2.42), again, we obtain:

$$
\begin{aligned}
\{f, g\}(\phi)= & <\phi,\left[R\left(a d f_{\phi}\right), d g_{\phi}\right]+\left[d f_{\phi}, R\left(a d g_{\phi}\right)\right]> \\
& -\frac{b}{2}\left(<R\left(D f_{\phi}\right), D g_{\phi}>+<D^{\prime} f_{\phi}, R\left(D^{\prime} g_{\phi}\right)>\right) \\
& +<P_{0}\left(a d f_{\phi}+b D f_{\phi}\right), d g_{E}>-<d f_{E}, P_{0}\left(\left(a d g_{\phi}+b D g_{\phi}\right)>,\right.
\end{aligned}
$$

which is the form of our brackets (2.13).

\section{iii) Trigonometric case}

This case is very similar to the elliptic case, and indeed is a limiting case of it. On the nodal curve $\Sigma$ the stable bundles of degree one are classified by their top exterior power in $\operatorname{Pic}^{0}(\Sigma)=\mathbb{C}^{*}[\mathrm{HuK}]$. The stable bundles, again, only have constant multiples of the identity as global automorphisms, so that again $\mathcal{N}_{\text {trig }}=\mathcal{M}_{\text {trig }} / A u t=\mathcal{M}_{\text {trig }}$, where $\mathcal{M}_{\text {trig }}$ is the open subset of the moduli corresponding to stable bundles.

The only new element one must deal with comes from the singularity of the curve. One can work on the desingularisation $\mathbb{P}_{1}$ of $\Sigma$. One-forms on $\Sigma$ are identified with oneforms on $\mathbb{P}^{1}$ with a simple pole at $0, \infty$ (and zero total residue), so that there is a global trivialisation of the cotangent bundle, given over $\mathbb{P}_{1}$ by the form $d \lambda / \lambda$; dually, the tangent bundle is spanned by $\lambda \frac{\partial}{\partial \lambda}$.

In a similar fashion, the Poisson structures on the total space $T$ of $\mathcal{O}(D)$ correspond to Poisson structures on the total space $\hat{T}$ of the lift of $\mathcal{O}(D)$ to $\mathbb{P}_{1}$, of the form:

$$
\frac{\lambda}{p(\lambda)}(a(\lambda)+b z p(\lambda))\left(\frac{\partial}{\partial \lambda} \wedge \frac{\partial}{\partial z}\right),
$$


where $p$ is a polynomial of degree $n$ vanishing at the lift of the divisor $D, a$ a polynomial of degree $n$ satisfying $a(0) / p(0)=\lim _{\lambda \rightarrow \infty} a(\lambda) / p(\lambda)$, and $b$ is a constant. There is then an $n+1$-dimensional space of such structures on $T$.

As for the elliptic case, one can take the corresponding family of Mukai structures, and reduce them by the action of $\operatorname{Pic}^{0}(\Sigma)$, obtaining:

Proposition (2.50) [HuK, HuMa] We have:

$$
\mathcal{M}_{\text {trig }}=\mathcal{N}_{\text {trig }}
$$

The Mukai Poisson structures on $\mathcal{N}_{\text {trig }}$ are equivalent those on $\mathcal{M}_{\text {trig }}$ given above.

\section{C) Divisor coordinates and Poisson surfaces}

We have given in the preceeding section (Poisson) embeddings of spaces $\mathcal{M} /$ Aut into spaces $\mathcal{N}$ of pairs $(S, L)$, where $S$ is a spectral curve lying in a surface $T$ and $L$ is a line bundle on the curve, (generically; in full generality, $L$ is a sheaf on $T$ supported on $S$ ) .

A line bundle on a curve can be represented by the divisor of zeroes of one of its holomorphic sections. If the degree of the line bundle is equal to the genus of the curve, the line bundle generically has a one-dimensional space of sections, and the map which to a divisor of degree $g$ associates the corresponding line bundle is a birational isomorphism between the $g$-th symmetric product $S P^{g}(\Sigma)$ of the curve and the variety $\operatorname{Pic}^{g}(\Sigma)$.

The curve, however, lies in a surface, and so the divisor not only determines the line bundle, but also the curve, at least partially, as the curve must pass through the points of the divisor.

Over an open set of $T$ containing all the spectral curves of an open set $U$ in our family, one can choose a line bundle $L_{0}$ such that the tensor product $L \otimes L_{0}$ is of degree $g$ over each spectral curve in $U$. Over an open set $V$ of pairs $(S, L)$, the line bundle $L \otimes L_{0}$ has a unique non-zero section, up to scale; this section vanishes over a divisor $\sum p_{\mu}$ of degree

$g$. This divisor can be thought of as an element of the $g$ th symmetric product $S P^{g}(T)$ of $T$, or as a length $g$ zero-dimensional subscheme of $T$.

One straightforward way to obtain the divisor is to consider the defining sequence (2.23) for $L$ :

$$
0 \rightarrow \pi^{*} E \otimes K_{\Sigma}^{*}(-D) \stackrel{\phi-\zeta \mathbb{I}}{\longrightarrow} \pi^{*} E \rightarrow L \rightarrow 0
$$

One can twist the fixed bundle $E$ so that it has up to scale a single section $\gamma$, which then by projection gives a section of $L$ which we will denote by $\gamma^{\prime}$. The section $\gamma^{\prime}$ vanishes when $\gamma$ lies in the image of $\phi-\zeta \mathbb{I}$. This gives the equation for the divisor

$$
(\phi-\zeta \mathbb{I})_{\mathrm{adj}} \gamma=0
$$

where the subscript adj denotes the classical adjoint (matrix of cofactors). 
As we have seen, the surfaces $T$ in which the spectral curves live have a family of Poisson structures, which are symplectic structures over open sets. These Poisson structures extend naturally to the symmetric product. The latter space is not smooth, as it is singular over the diagonal. It does have a natural desingularisation, the Hilbert scheme $H_{i l b}(T)$ of 0 -dimensional ideals of length $g$, and the Poisson structures lift to $H i l b^{g}(T)$ $[\mathrm{B}]$.

Proposition (2.52) For each Poisson structure in our family on $T$, taking the corresponding Mukai structure, the map

$$
\begin{aligned}
\mathcal{N} & \rightarrow \operatorname{Hilb}^{g}(T), \\
(S, L) & \mapsto \sum p_{\mu}
\end{aligned}
$$

is Poisson.

Proof: The proof hinges on the fact that the Mukai structures at $(S, L)$ are independent of the resolution chosen of the sheaf $L$, and so one can choose a convenient resolution. Let us suppose we are at a generic point, at which $S$ is a smooth curve and $L$ a line bundle over $S$. If we extend $L$ to a neighbourhood of the curve, we have

$$
\left.0 \rightarrow L \otimes N_{S}^{*} \stackrel{\operatorname{det}(\phi-z \mathbb{I})}{\longrightarrow} \quad L \rightarrow L\right|_{S} \rightarrow 0
$$

as a resolution. Taking $\operatorname{Hom}\left(\cdot, L \otimes K_{T}\right)$ of this sequence, we have, that $T^{*} V$ is the first hypercohomology of the sequence

$$
\mathcal{O}_{S} \otimes K_{T} \rightarrow N_{S} \otimes K_{T}
$$

supported over $S$; the map is the zero map. $T^{*} V$ is then the sum

$$
T^{*} V=H^{1}\left(S, \mathcal{O}_{S} \otimes K_{T}\right) \oplus H^{0}\left(S, K_{S}\right),
$$

using the isomorphism $K_{T} \simeq K_{S} \otimes N_{S}^{*}$, and dually,

$$
T V=H^{0}\left(S, N_{S}\right) \oplus H^{1}\left(S, \mathcal{O}_{S}\right)
$$

The pairing between $T^{*} V$ and $T V$ is given by Serre duality: $H^{1}\left(S, \mathcal{O}_{S} \otimes K_{T}\right)$ is the dual of $H^{0}\left(S, N_{S}\right)$ and $H^{1}\left(S, \mathcal{O}_{S}\right)$ is the dual of $H^{0}\left(S, K_{S}\right)$. The Poisson tensor applied to two covectors $(\alpha, \beta),\left(\alpha^{\prime}, \beta^{\prime}\right)$ is then given by

$$
<\alpha, \theta \beta^{\prime}>-<\alpha^{\prime}, \theta \beta>
$$

The proof is then a matter of writing out the pairings explicitly. Let $\sum_{\mu} p_{\mu}$ be the divisor corresponding to $L$, and suppose for simplicity that the points $p_{\mu}$ are distinct. Let $\rho=0$ be the defining equation for the curve $S$, and $\sigma$ be the section of $L \otimes L_{0}$, so that the $p_{\mu}$ are defined by the simultaneous vanishing of $\rho, \sigma$. On $T V$, variations of the curve $S$ correspond to sections $v$ of the normal bundle $H^{0}\left(S, N_{S}\right)$, while variations of the line bundle are given 
by the cocycle $\dot{\sigma} / \sigma$ defined on punctured discs surrounding the $p_{\mu}$, so that the differential $(\dot{\sigma} / \sigma, v) \mapsto \dot{p}_{\mu}$ of the map $(S, L) \rightarrow \sum_{\mu} p_{\mu}$ is given by the two conditions

$$
\begin{aligned}
d \rho\left(v\left(p_{\mu}\right)\right) & =d \rho\left(\dot{p}_{\mu}\right), \\
\dot{\sigma} & =-d \sigma\left(\dot{p}_{\mu}\right) .
\end{aligned}
$$

If $f, g$ are functions on $\mathrm{Hilb}^{g}(T)$, let $F, G$ denote the corresponding functions on $\mathcal{N}$. The differential $d F$ at a point $(S, L)$ is represented by a pair $\left(\tau^{F}, \omega^{F}\right)$ in the sum $(2.54)$. Representing $\tau^{F}$ by cocycles $\tau_{\mu}^{F}$ with values in $K_{T}$ on punctured disks around the $p_{\mu}$, we have from (2.57)

$$
\tau_{\mu}^{F}=d_{\mu} f \wedge \frac{d \sigma}{\sigma}
$$

while

$$
\omega^{F}\left(p_{\mu}\right)=\left.d_{\mu} f\right|_{S} .
$$

Evaluating the Poisson bracket on $F, G$.

$$
\begin{aligned}
\{F, G\} & =<\theta \tau^{F}, \omega^{G}>-<\theta \tau^{G}, \omega^{F}> \\
& =\sum_{\mu} \operatorname{res}_{\mu}\left(<\theta, d_{\mu} f \wedge \frac{d \sigma}{\sigma}>\left.d_{\mu} g\right|_{S}-<\theta, d_{\mu} g \wedge \frac{d \sigma}{\sigma}>\left.d_{\mu} f\right|_{S}\right), \\
& =\sum_{\mu}<\theta, d_{\mu} f \wedge d_{\mu} g>
\end{aligned}
$$

which is the Poisson bracket of $f, g$.

We note that for each Poisson structure in the family, $H i l b^{g}(T)$ is symplectic over the open set of ideals whose support is disjoint from the zero divisor $P$ of the Poisson structure on $T$. The map which to a pair $(S, L)$ associates its divisor is generically immersive if one fixes $S$, or, on the level of tangent spaces, is generically injective on the summand $H^{1}(S, \mathcal{O})$ of $T V$. As the map is Poisson, this tells us that the map $\mathcal{N} \rightarrow H_{i l b}(T)$ is an isomorphism on the level of symplectic leaves.

Reduction to $\operatorname{sl}(r)$.

In two of the cases which concerned us, the elliptic and trigonometric, the more usual phase spaces consist of $s l(r)$ or $S l(r)$-valued functions, and, as we saw, we could obtain these phase spaces by reduction. This was done by fixing the highest exterior power of the bundle $E$, and then shifting $\phi$ either additively or multiplicatively by a constant so that it is traceless or of fixed determinant. This has a good interpretation in the elliptic case, when $\Sigma$ is a group. The top exterior power of $E$ is represented, up to a constant, by the sum $\sum_{\mu} \pi\left(p_{\mu}\right)$. Let us fix a (linear) coordinate $\lambda$ on the base elliptic curve, and take a fiber coordinate $z$ in $T$, such that the Poisson tensor on $T$ is given by $(a(\lambda)+b z)\left(\frac{\partial}{\partial \lambda} \wedge \frac{\partial}{\partial z}\right)$. Fixing the top exterior power amounts to fixing $\sum_{\mu} \lambda_{\mu}$. Taking $\sum_{\mu} \lambda_{\mu}$ as a Hamiltonian, we get flows $\dot{\lambda}_{\mu}=0, \dot{z}_{\mu}=a\left(\lambda_{\mu}\right)+b z_{\mu}$, which of course are compatible with the flows for $\phi$. We can normalise using these flows; one generically valid normalisation is $\sum_{\mu} z_{\mu}=0$. 


\section{D) INTEGRABLE Systems}

There is a family of commuting Hamiltonian systems defined on our different spaces, which indeed is integrable at levels (b), (c) for all our Poisson structures. At level (a), it is given by Hamiltonians of the form

$$
F_{\omega, n}(\phi)=\operatorname{res}\left(\omega \operatorname{tr}\left(\phi^{n}\right)\right)
$$

where $\omega$ is a scalar cocycle; in other words, the Hamiltonians are the coefficients of the spectrum of $\phi$. At level (b), the Lagrangian leaves are given by fixing the spectral curve; in other words, they are the fibers of the projection

$$
(S, L) \mapsto S
$$

Corresponding to this, on the level of tangent spaces to $\mathcal{N}$, one has from (2.55) an exact sequence

$$
0 \rightarrow H^{1}(S, \mathcal{O}) \rightarrow T \mathcal{N} \rightarrow H^{0}\left(S, N_{S}\right) \rightarrow 0 .
$$

Indeed, deformations of line bundles on a spectral curve are given by $H^{1}(S, \mathcal{O})$, while deformations of the spectral curve are given by sections of the normal bundle. It is quite easy to see that the foliation is Lagrangian, under the Mukai bracket. Indeed, dually to (2.61), we have:

$$
0 \rightarrow H^{1}\left(S, K_{T}\right) \rightarrow T^{*} \mathcal{N} \rightarrow H^{0}\left(S, K_{S}\right) \rightarrow 0 .
$$

Functions on the base of (2.60), lifted to $\mathcal{N}$, have their differentials in the summand $H^{1}\left(S, K_{T}\right)$; under the action of the Poisson tensor $\Lambda$, this gets mapped to $H^{1}(S, \mathcal{O})$. Referring to $(2.56)$ for a pair of differentials $d F=(\alpha, 0), d G=\left(\alpha^{\prime}, 0\right)$ of functions $F, G$ lifted from the base, we have

$$
\{F, G\}=0,
$$

so that the fibration is indeed Lagrangian. One can also see which of these functions are Casimirs: they correspond to the kernel of the map $H^{1}\left(S, K_{T}\right) \rightarrow H^{1}(S, \mathcal{O})$ given by multiplication by the Poisson tensor $\theta$ on $T$. This is part of a long exact sequence

$$
0 \rightarrow H^{0}\left(S, K_{T}\right) \rightarrow H^{0}(S, \mathcal{O}) \rightarrow H^{0}(P, \mathcal{O}) \rightarrow H^{1}\left(S, K_{T}\right) \rightarrow H^{1}(S, \mathcal{O}) \rightarrow 0
$$

where $P$ is the divisor of $\theta$ on the curve $S$. The image $\delta\left(H^{0}(P, \mathcal{O})\right) \subset H^{1}\left(S, K_{T}\right)$ correspond to the differentials of the defining equations of the spectral curve at $P$, and so these are the Casimirs. In other words, the symplectic leaves of the Poisson structure at level (b) are given by fixing the spectral curves at $P$. We note that as $\theta$ varies, $P$ moves all over the spectral curve, and so:

Proposition (2.67) Fixing the values of the Casimirs for all the Poisson structures in our families is tantamount to fixing the spectral curve $S$ : the joint level sets of the Casimirs is then an open set of the Jacobian of $S$.

This proposition and the integrability of the system gives us Theorem 2 of the introduction. We note that at level (c) the lagrangian leaves in $H i l b^{g}(T)$ are given simply by constraining the points to lie on the curves $S$, so that the leaves are $H i l b^{g}(S) \subset H i l b^{g}(T)$. 


\section{Nijenhuis coordinates.}

\section{A) Privileged coordinate systems}

Recapitulating, we have established a chain of maps between:

(a) Certain spaces $\mathrm{M}$ of matrix-valued functions on the line (times a curve, in the trigonometric and elliptic cases), equipped with a family of $r$-matrix-type brackets;

(b) Spaces of pairs $(S, L)$ of spectral curves $S$ and sheaves $L$ supported on these curves, equipped with a family of Mukai brackets;

(c) Spaces of length $g$ 0-dimensional ideals on a surface, equipped with a family of Poisson brackets, induced from a similar family on the surface.

The passage from (a) to (b) is obtained by thinking of the matrix valued function as a meromorphic endomorphism of a fixed vector bundle $E$, and taking then its spectral curve $S$ and its associated (dual) eigensheaf $L$.

To summarise, we state the

Theorem (3.1) On levels (a), (b), (c), we have linear families of Poisson structures of dimensions $(n+3)$ in the rational case, $(n+1)$ in the elliptic and trigonometric cases. In each case, the families on the three levels can be identified, so that the maps relating levels (a), (b) and (c) are Poisson for each structure in the family. The map from level (a) to (b) takes a quotient by a group of automorphisms, which is trivial in the elliptic and trigonometric cases; the map from level (b) to (c) is generically immersive on symplectic leaves.

This is most of Theorem 1 of the introduction. In case (c), because we are dealing with what is in essence a symmetric product of the surface, there are natural sets of coordinates which can be exploited. On a surface, Poisson structures are simply sections of the line bundle $K_{T}^{*}$, and so the quotient of any two of them is a meromorphic function. Thus, if we choose Darboux coordinates $\lambda, z$ for one of the structures in our family, so that the Poisson tensor is $\frac{\partial}{\partial \lambda} \wedge \frac{\partial}{\partial z}$, the other Poisson structures are $f(\lambda, z) \frac{\partial}{\partial \lambda} \wedge \frac{\partial}{\partial z}$, for $f$ a meromorphic function in a linear system. On the level of the symmetric product, then, the induced coordinates $\lambda_{\mu}, z_{\mu}$ give a Poisson tensor of the form

$$
\Lambda=\sum_{\mu} f\left(\lambda_{\mu}, z_{\mu}\right) \frac{\partial}{\partial \lambda_{\mu}} \wedge \frac{\partial}{\partial z_{\mu}} .
$$

Let us give the following definition of Nijenhuis coordinates: suppose that one has two Poisson tensors

$$
\Lambda_{1}, \Lambda_{2}: T^{*} M \rightarrow T M,
$$

for some manifold $M$, and suppose that $\Lambda_{2}$ is non-degenerate. We say that coordinates $f_{1}, \ldots f_{n}$ on $M$ are Nijenhuis coordinates if their differentials $d f_{i}$ are eigenvectors of $\Lambda_{2}^{-1} \Lambda_{1}$. (This is the definition given, e.g. in [FP]; in the next subsection we show how it is related to that of $[\mathrm{N}]$.) One has: 
Proposition (3.4) The coordinates $\lambda_{\mu}, z_{\mu}$ are Nijenhuis coordinates in case (c) for any pair of Poisson structures in our family.

One can ask what one does when the Poisson tensor has a kernel, for example at level (b). One frequent way of dealing with a map which has a kernel is to consider its graph instead, and generalise from there. Let us consider in $T^{*} M \times T^{*} M$ the space $V$ :

$$
\left\{\left(v_{1}, v_{2}\right) \mid \Lambda_{1}\left(v_{1}\right)=\Lambda_{2}\left(v_{2}\right)\right\},
$$

and say that $v$ is an eigenvector of the pair $\Lambda_{1}, \Lambda_{2}$ if

$$
\Lambda_{1}\left(\alpha_{1} v\right)=\Lambda_{2}\left(\alpha_{2} v\right),
$$

for some constants $\alpha_{1}, \alpha_{2}$, i.e., if $\left(\alpha_{1} v, \alpha_{2} v\right)$ lies in $V$. With this convention, one can define Nijenhuis coordinates in the degenerate case, as in the non-degenerate one. One has, for $\mathcal{N}$ in the cases we have considered,

Proposition(3.5) For any pair of Poisson structures $\Lambda_{1}, \Lambda_{2}$ in our family, if we complete the functions $\lambda_{\mu}, z_{\mu}$ by Casimir functions for either $\Lambda_{1}$ or $\Lambda_{2}$ to a coordinate system, we obtain Nijenhuis coordinates on $\mathcal{N}$.

\section{B) Definitions of Nijenhuis coordinates.}

We now explain briefly how our definition of Nijenhuis coordinates, which we believe is more adapted to a multi-Hamiltonian (as opposed to bi-Hamiltonian) situation, is related to the classical definition of $[\mathrm{N}]$; see also [Mg2], [GZ]. Given two compatible Poisson structures

$$
\Lambda_{1}, \Lambda_{2}: T^{*} M \rightarrow T M,
$$

with the second non-degenerate, the classical Nijenhuis coordinates $[\mathrm{N}]$ are defined as the eigenvalues of $\Lambda_{1}^{-1} \Lambda_{2}$. We shall see here that a more suitable notion when dealing with several Poisson structures is to consider instead coordinate functions whose differentials are eigenvectors of $\Lambda_{1}^{-1} \Lambda_{2}$.

Let us suppose that the tensor $\Lambda_{1}^{-1} \Lambda_{2}$ can be diagonalised in a neighbourhood of a point with eigenvector 1-forms $\omega_{i}$ and eigenvalues $\rho_{i}$. A first remark is

Lemma (3.7) Let $\rho_{i} \neq \rho_{j}$. Then $<\omega_{i}, \Lambda_{1}\left(\omega_{j}\right)>=<\omega_{i}, \Lambda_{2}\left(\omega_{j}\right)>=0$.

Proof: Recall that the Poisson tensors are skew adjoint. We then have

$$
\begin{aligned}
\rho_{i}<\omega_{i}, \Lambda_{1}\left(\omega_{j}\right)> & =<\Lambda_{1}^{-1} \Lambda_{2}\left(\omega_{i}\right), \Lambda_{1}\left(\omega_{j}\right)> \\
& =-<\omega_{j}, \Lambda_{2}\left(\omega_{i}\right)> \\
& =<\omega_{i}, \Lambda_{2}\left(\omega_{j}\right)> \\
& =-<\Lambda_{1}^{-1} \Lambda_{2}\left(\omega_{j}\right), \Lambda_{1}\left(\omega_{i}\right)> \\
& =-\rho_{j}<\omega_{j}, \Lambda_{1}\left(\omega_{i}\right)> \\
& =\rho_{j}<\omega_{i}, \Lambda_{1}\left(\omega_{j}\right)>
\end{aligned}
$$


The proof for $\Lambda_{2}$ is similar.

The nondegeneracy of the forms then forces the eigenvectors to come in pairs, one pair for each eigenvalue. We will suppose, as a genericity constraint, that otherwise the eigenvectors are distinct. Rescaling the $\omega_{i}$ and renumbering the eigenvalues, we can then write the Poisson tensors as

$$
\begin{aligned}
& \Lambda_{1}=\sum_{i=1}^{n} \omega_{2 i-1}^{*} \wedge \omega_{2 i}^{*} \\
& \Lambda_{2}=\sum_{i=1}^{n} \rho_{i} \omega_{2 i-1}^{*} \wedge \omega_{2 i}^{*}
\end{aligned}
$$

and, dually, the symplectic forms as

$$
\begin{aligned}
& \Omega_{1}=\sum_{i=1}^{n} \omega_{2 i-1} \wedge \omega_{2 i} \\
& \Omega_{2}=\sum_{i=1}^{n} \rho_{i}^{-1} \omega_{2 i-1} \wedge \omega_{2 i} .
\end{aligned}
$$

The next step is to show that one can replace the $\omega_{2 i-1} \wedge \omega_{2 i}$ by $d x_{2 i-1} \wedge d x_{2 i}$, for suitable coordinate functions. To do this, it will suffice to show that $d\left(\omega_{2 i-1} \wedge \omega_{2 i}\right)=0$. Indeed, Frobenius' theorem then tells us that the distribution generated by $\omega_{2 i-1} \wedge \omega_{2 i}$ is integrable, and so one has functions $y_{2 i-1}, y_{2 i}$ such that $\omega_{2 i-1} \wedge \omega_{2 i}=f d y_{2 i-1} \wedge d y_{2 i}$, for some function $f$. But then, since $d\left(\omega_{2 i-1} \wedge \omega_{2 i}\right)=0$, one finds that $f$ can only depend on $y_{2 i-1}, y_{2 i}$, and so one choose $x_{2 i-1}=y_{2 i-1}, x_{2 i}=\int f d y_{2 i}$.

LEMMA $(3.11) d\left(\omega_{2 j-1} \wedge \omega_{2 j}\right)=0, j=1, . ., n$.

ProOf: We use the compatibility of the Poisson structures, more precisely, that $\Lambda_{1}-a \Lambda_{2}$ is a Poisson structure for all values of $a$. This tells us that the forms

$$
\Omega_{a}=\sum_{i=1}^{n}\left(1-a \rho_{i}\right)^{-1} \omega_{2 i-1} \wedge \omega_{2 i}
$$

are closed for all values of $a$. Expanding in powers of $a$ : $\left(1-a \rho_{i}\right)^{-1}=1+a \rho_{i}+\left(a \rho_{i}\right)^{2}+\ldots$ and taking derivatives in $a$ at $a=0$ tells us that for all polynomials $p$, the forms

$$
{ }_{p} \Omega=\sum_{i=1}^{n} p\left(\rho_{i}\right) \omega_{2 i-1} \wedge \omega_{2 i}
$$

are also closed. Now, at a given point $x$, choose $p$ so that $p\left(\rho_{j}(x)\right)=1, p\left(\rho_{i}(x)\right)=0$ for $i \neq j$, and $p^{\prime}\left(\rho_{i}(x)\right)=0$ for all $i$. At $x$, the exterior derivative of ${ }_{p} \Omega$ evaluates to $d\left(\omega_{2 j-1} \wedge \omega_{2 j}\right)$ which must then vanish. As the choice of point is arbitrary, we are done. 
Our symplectic forms are now:

$$
\begin{aligned}
& \Omega_{1}=\sum_{i=1}^{n} d x_{2 i-1} \wedge d x_{2 i} \\
& \Omega_{2}=\sum_{i=1}^{n} \rho_{i}^{-1} d x_{2 i-1} \wedge d x_{2 i}
\end{aligned}
$$

The last step is to remark that the fact that $\Omega_{2}$ is closed tells us that $\rho_{i}$ depends on $x_{2 i-1}, x_{2 i}$ only, giving

$$
\begin{aligned}
& \Omega_{1}=\sum_{i=1}^{n} d x_{2 i-1} \wedge d x_{2 i}, \\
& \Omega_{2}=\sum_{i=1}^{n} \rho_{i}^{-1}\left(x_{2 i-1}, x_{2 i}\right) d x_{2 i-1} \wedge d x_{2 i} .
\end{aligned}
$$

We now have a normal form, and note that the $x_{i}$ are Nijenhuis coordinates as their differentials are eigenvectors of the Nijenhuis operator. We have seen that under some mild non-degeneracy conditions, these coordinates exist, and come in pairs, one pair per eigenvalue. We note that the pair is defined only up to a volume preserving diffeomorphism of the plane. One canonical choice for the first coordinate of the pair, assuming some nondegeneracy, would simply be the function $\rho_{i}\left(x_{2 i-1}, x_{2 i}\right)$, giving the classical definition of Nijenhuis coordinate as an eigenvalue: the classical definition picks out one coordinate from amongst all of the functions of $x_{2 i-1}, x_{2 i}$. On the other hand, with the modified definition, if we have an $n$-dimensional family of Poisson structures, one can show that with some non-degeneracy assumptions one has normal forms

$$
\begin{aligned}
\Omega_{1}= & \sum_{i=1}^{n} d x_{2 i-1} \wedge d x_{2 i} \\
\Omega_{2}= & \sum_{i=1}^{n} \rho_{2, i}^{-1}\left(x_{2 i-1}, x_{2 i}\right) d x_{2 i-1} \wedge d x_{2 i} \\
\Omega_{3}= & \sum_{i=1}^{n} \rho_{3, i}^{-1}\left(x_{2 i-1}, x_{2 i}\right) d x_{2 i-1} \wedge d x_{2 i} \\
& \ldots \\
\Omega_{n}= & \sum_{i=1}^{n} \rho_{n, i}^{-1}\left(x_{2 i-1}, x_{2 i}\right) d x_{2 i-1} \wedge d x_{2 i}
\end{aligned}
$$

so that the coordinates $x_{i}$ are Nijenhuis coordinates for the whole family, i.e., independently of the pair $\Omega_{i}, \Omega_{j}$ one chooses.

\section{More explicit formulae: the rational case.}


We now explain how the above theorems give coordinate systems which are quite tractable computationally, and so allow fairly explicit integration of the systems. We will simplify, and only consider the rational case. The necessary extra computations required to deal with the elliptic and trigonometric cases are given in $[\mathrm{HuK}]$.

Recall that our level (a) phase space, consisting of matricial polynomials $\phi(\lambda)$ of degree at most $n$, reduces at level (b) to a space of generically smooth spectral curves defined by

$$
\operatorname{det}(\phi-z \mathbb{I})=0
$$

and (generically) line bundles $L$ defined by

$$
0 \rightarrow \mathcal{O}^{\oplus r} \otimes \mathcal{O}(-n) \stackrel{\phi-z \mathbb{I}}{\longrightarrow} \mathcal{O}^{\oplus r} \rightarrow L \rightarrow 0
$$

The divisor coordinates at level (c) are obtained by considering the points on $S$ where the projection to $L$ of a standard section of $\mathcal{O}^{\oplus r}$ vanishes. We choose the section $\gamma=$ $(1,0, \ldots, 0)^{T}$ of $\mathcal{O}^{\oplus r}$; as a section of $L$, it vanishes where it lies in the image of $\phi-z \mathbb{I}$, that is, when

$$
(\phi-z \mathbb{I})_{\operatorname{adj}} \gamma=0,
$$

where the subscript denotes taking the matrix of cofactors (classical adjoint). If we assume that $\phi$ is normalised to have its leading order term diagonal, this gives $r-1$ fixed (for a given spectral curve) points over $z=\infty$, and $g=\operatorname{genus}(S)$ points $p_{\mu}=\left(\lambda_{\mu}, z_{\mu}\right)$ over the rest of the curve. It is these points that provide the coordinates. Another way of obtaining these points, following [Sc, Ge] is by noting that $\lambda_{\mu}$ are the $\lambda$-coordinates of points for which $V$ is not a cyclic vector for $\phi(\lambda)$, and so a defining equation for $\lambda_{\mu}$ is

$$
\operatorname{det}\left(V, \phi(\lambda)(V), \phi(\lambda)^{2}(V), \ldots, \phi(\lambda)^{r-1}(V)\right)=0
$$

One can then obtain the $z_{\mu}$ as follows: one chooses another vector $W$ and sets

$$
\begin{aligned}
& P(\lambda)=\operatorname{det}\left(W, V, \phi(\lambda)(V), \phi(\lambda)^{2}(V), \ldots, \phi(\lambda)^{r-3}(V), \phi(\lambda)^{r-2}(V)\right), \\
& R(\lambda)=\operatorname{det}\left(W, V, \phi(\lambda)(V), \phi(\lambda)^{2}(V), \ldots, \phi(\lambda)^{r-3}(V), \phi(\lambda)^{r-1}(V)\right),
\end{aligned}
$$

One chooses $W$ so that $P\left(\lambda_{\mu}\right)$ is non-vanishing. One then has:

$$
z_{\mu}=(-1)^{r}\left[\frac{R\left(\lambda_{\mu}\right)}{P\left(\lambda_{\mu}\right)}-\operatorname{tr}\left(\phi\left(\lambda_{\mu}\right)\right)\right]
$$

This then gives us the Nijenhuis coordinates in a fairly explicit way. We now consider the Poisson structures. As noted above, at level (c), we have a family of Poisson structures of the form

$$
\Lambda_{a, b}=\sum_{\mu}\left(a\left(\lambda_{\mu}\right)+b z_{\mu}\right)\left(\frac{\partial}{\partial \lambda_{\mu}} \wedge \frac{\partial}{\partial z_{\mu}}\right),
$$


where $a$ is a polynomial of degree at most $(n+1)$ and $b$ a constant. Choosing a polynomial $\alpha$ of degree at most $n$, one can consider the two-dimensional linear subfamily (pencil) of Poisson structures

$$
\Lambda_{c, c^{\prime}}=\sum_{\mu}\left(c \lambda_{\mu}-c^{\prime}\right) \alpha\left(\lambda_{\mu}\right)\left(\frac{\partial}{\partial \lambda_{\mu}} \wedge \frac{\partial}{\partial z_{\mu}}\right),
$$

where $c, c^{\prime}$ are constants. Notice then that the $\lambda_{\mu}$ are (classical) Nijenhuis coordinates for the Poisson structures $\Lambda_{1,0}, \Lambda_{0,1}$. The Casimirs for the $\Lambda_{1, c}$ structure include the intersections of the spectral curve with $\lambda=c$, and so are the $z$-coefficients of

$$
\operatorname{det}(\phi(c)-z \mathbb{I})=0
$$

These are polynomials of degree at most $n r$ in $c$, giving our generalisation of the Gel'fandZakharevich theorem. We note that as $c$ varies, the whole spectral curve is swept out, and so one indeed has the full set of Hamiltonians from taking the union of the Casimirs over all $c$.

As shown in $[\mathrm{AHH}]$, the flows corresponding to the Poisson structure $\Lambda_{a, b}$ and the Hamiltonian $H_{i}$ (choosing a basis $H_{1}, . ., H_{k}$ for the space of Hamiltonians) can be obtained as follows, through a fairly standard generating function argument. Fixing the Hamiltonians fixes the spectral curve, and so determines $z$ as a function of $\lambda: z=z\left(\lambda, H_{1}, \ldots, H_{k}\right)$. Choosing a base point $\lambda_{0}$ on the spectral curve, we set

$$
\begin{aligned}
F\left(\lambda_{1}, \ldots, \lambda_{g}, H_{1}, \ldots, H_{k}\right) & =\sum_{\mu} \int_{\lambda_{0}}^{\lambda_{\mu}} b^{-1} \ln \left(a(\lambda)-b z\left(\lambda, H_{1}, \ldots, H_{k}\right)\right) d \lambda, \text { for } b \neq 0, \\
& \left.=\sum_{\mu} \int_{\lambda_{0}}^{\lambda_{\mu}}(a(\lambda))^{-1} z\left(\lambda, H_{1}, \ldots, H_{k}\right)\right) d \lambda, \text { for } b=0 .
\end{aligned}
$$

The linearising coordinates of the flows are given by

$$
Q_{i}=\frac{\partial F}{\partial H_{i}}=\sum_{\mu} \int_{\lambda_{0}}^{\lambda_{\mu}}(a(\lambda)-b z)^{-1} \frac{\partial \lambda}{\partial H_{i}} d z
$$

One can show that these are sums of Abelian integrals.

One then has explicit formulae for the flows. In solving a particular system, it is then a matter of writing out the separating variables in terms of the variables at hand. We give briefly the example of the Neumann oscillator, describing motion on the sphere $\sum_{i=1}^{n} x_{i}^{2}=1$ under the influence of a quadratic potential $\sum_{i=1}^{n} \alpha_{i} x_{i}^{2}$, with conjugate momentum variables $y_{i}$ satisfying $\sum_{i=1}^{n} x_{i} y_{i}=0$. (Compare $[\mathrm{P}]$ ). One has, at the loop algebra level, the following parametrization:

We set

$$
a(\lambda)=\prod_{i=1}^{n}\left(\lambda-\alpha_{i}\right), \quad a_{j}(\lambda)=\prod_{i=1, i \neq j}^{n}\left(\lambda-\alpha_{i}\right)
$$


and set

$$
\phi(\lambda)=a(\lambda)\left(\begin{array}{cc}
0, & -1 / 2 \\
0, & 0
\end{array}\right)+\left(\begin{array}{cc}
-\sum_{i=1}^{n} x_{i} y_{i} a_{i}(\lambda), & -\sum_{i=1}^{n} y_{i}^{2} a_{i}(\lambda) \\
\sum_{i=1}^{n} x_{i}^{2} a_{i}(\lambda), & \sum_{i=1}^{n} x_{i} y_{i} a_{i}(\lambda)
\end{array}\right)
$$

The Hamiltonian for the system is

$$
\operatorname{Res}_{\infty}\left(\operatorname { d e t } \left(\lambda \phi(\lambda) / a(\lambda)^{2}\right.\right.
$$

The separating coordinates are simply the roots $\lambda_{\mu}$ of

$$
\sum_{i=1}^{n} x_{i}^{2} a_{i}(\lambda)=0
$$

(these are the classical ellipsoidal coordinates) together with

$$
z_{\mu}=\sum_{i=1}^{n} x_{i} y_{i} a_{i}\left(\lambda_{\mu}\right)
$$

\section{References}

[At] M. Atiyah, Vector bundles over an elliptic curve, Proc. Lond. Math. Soc 7, 414-452 (1957).

[AHH] M.R. Adams,J. Harnad, and J. Hurtubise, Darboux coordinates and Liouville-Arnold integration in loop algebras, Comm. Math. Phys. 155 , no. 2, 385-413 (1993).

[AHP] M.R. Adams, J. Harnad, and E. Previato, Isospectral Hamiltonian flows in finite and infinite dimensions I. Generalised Moser systems and moment maps into loop algebras, Comm. Math. Phys. 117, no. 3, 451-500 (1988).

[AvM] M. Adler and P. van Moerbeke, Completely integrable systems, Euclidean Lie algebras, and curves, Adv. in Math. 38, no. 3, 267-317 (1980); Linearization of Hamiltonian systems, Jacobi varieties and representation theory, ibid. 38, no. 3, 318-379 (1980).

[B] A.Beauville, Variétés Kählériennes dont la première classe de Chern est nulle. Jour. Diff.Geom. 18 755-782 (1983).

[Bo] F. Bottacin, Symplectic geometry on moduli spaces of stable pairs, Ann. Sci. Ecole Norm. Sup. (4) 28, no. 4, 391-433 (1995).

[FP] G. Falqui and M. Pedroni, Separation of variables for bi-Hamiltonian systems, 47 pages, preprint.

[FT] L.D. Faddeev and L.A. Takhtajan, Hamiltonian methods in the theory of solitons, eds., Springer-Verlag, Berlin, 1987. 
[Ge] M.I. Gekhtman, Separation of variables in the classical SL $(N)$ magnetic chain. Comm. Math. Phys. 167, no. 3, 593-605 (1995).

[GZ] I.M. Gel'fand and I. Zakharevich, On the local geometry of a bi-Hamiltonian structure in: The Gel'fand Mathematical Seminars 1990-1992 (L. Corwin et al. eds.) Birkhauser, Boston 1993, pp. 51-112.

[HHu] J. Harnad and J. Hurtubise, Generalised tops and moment maps into loop algebras, J. Math. Phys. 37, no. 7, 1780-1787 (1991).

[Hi1] N.J. Hitchin, The self-duality equations on a Riemann surface, Proc. London Math. Soc. (3) 55, no. 1, 59-126 (1987).

[Hi2] N.J. Hitchin, Stable bundles and integrable systems, Duke Math. J. 54, no. 1, 91-114 (1987).

[Hu] J. Hurtubise, Integrable systems and algebraic surfaces, Duke Math. J. 83, no. 1, 19-50 (1996).

$[\mathrm{HuK}]$ J. Hurtubise and M. Kjiri, Separating coordinates for the generalized Hitchin systems and the classical r-matrices Commun. Math. Phys 210, 521-540 (2000).

[HuMa] J. Hurtubise and E. Markman, Surfaces and the Sklyanin bracket, 19 p., to appear in Commun. Math. Phys. math.AG/0107010.

[Ma] E. Markman, Spectral curves and integrable systems, Compositio Math. 93, 255-290, (1994).

[Mg1] F. Magri, "Eight lectures on Integrable Systems" Integrability of nonlinear systems (Pondicherry, 1996), Lecture Notes in Phys., 495 Springer, Berlin-Heidelberg 1997, $256-296$.

[Mg2] F. Magri, "Geometry and Soliton Equations" La mécanique analytique de Lagrange et son héritage, Atti Acc. Sci. Torino Suppl. 124, 181-209 (1990).

[Mo] J. Moser, "Geometry of Quadrics and Spectral Theory", The Chern Symposium, Berkeley, June 1979, 147-188, Springer, New York, 1980.

$[\mathrm{Mu}]$ S. Mukai, Symplectic structure of the moduli space of sheaves on an abelian or K3 surface. Invent. Math. 77, no. 1, 101-116 (1984).

[N] A. Nijenhuis, $X_{n-1}$-forming sets of eigenvectors. Nederl. Akad. Wetensch. Proc. Ser. A. 54 - Indagationes Math. 13, 200-212 (1951).

[NS] M. Narasimhan and C.S. Seshadri, Stable and Unitary vector bundles on a compact Riemann surface, Annals of Math 82, 540-567 (1965).

[P] M. Pedroni, Bi-Hamiltonian aspects of the separability of the Neumann system 12 pages, nlin.SI/0202023.

[RS1] A.G. Reiman and M.A. Semenov-Tian-Shansky, Reduction of Hamiltonian systems, affine Lie algebras and lax equations I, II, Invent. Math. 54, no. 1, 81-100 (1979); 
ibid. 63, no. 3, 423-432 (1981).

[RS2] A.G. Reiman and M.A. Semenov-Tian-Shansky, Integrable Systems II, chap.2, in "Dynamical Systems VII", Encyclopaedia of Mathematical Sciences, vol 16., V.I. Arnold and S.P.Novikov, eds., Springer-Verlag, Berlin, 1994.

[Sc] D.R.D. Scott, Classical functional Bethe ansatz for $S L(N)$ :separation of variables for the magnetic chain, J. Math. Phys. 35, 5831-5843 (1994).

[Sk1] E.K. Sklyanin, On the complete integrability of the Landau-Lifschitz equation, LOMI preprint E-3-79, (1979).

[Sk2] E.K. Sklyanin, Poisson structure of a periodic classical XYZ-chain, J. Sov. Math. ,46, 1664-1683 (1989) .

[Ty] A.N. Tyurin, Symplectic structures on the moduli spaces of vector bundles on algebraic surfaces with $p_{g}>0$. (Russian) Izv. Akad. Nauk SSSR Ser. Mat. 52, no. 4, 813852, 896 (1988); translation in Math. USSR-Izv. 33, no. 1, 139-177 (1989).

J.Harnad: Department of Mathematics and Statistics, Concordia University, and Centre de Recherches Mathématiques. (harnad@crm.umontreal.ca)

J. C. Hurtubise: Department of Mathematics and Statistics, McGill University, and Centre de Recherches Mathématiques.(hurtubis@crm.umontreal.ca) 